\title{
Microdureza de caries incipientes artificiales infiltradas con resinas de baja viscosidad antes y después del termociclado
}

\section{Microhardness of artificial white-spot lesions infiltrated with low viscosity resins before and after thermocycling}

\section{Zamorano Pino X*, Valenzuela Aránguiz V*, Vial Prado C*, Vidal Tardón M*}

\section{RESUMEN}

Introducción: En Odontología Mínimamente Invasiva, el procedimiento denominado Infiltración de caries, pretende detener la progresión de las caries incipientes mediante el sellado de las microporosidades del esmalte afectado. Este tratamiento se realiza con la aplicación de resinas de baja viscosidad. El objetivo de este trabajo fue evaluar la microdureza superficial de caries incipientes artificiales infiltradas con resinas de baja viscosidad antes y después del proceso de termociclado.

Metodología: Se provocaron caries artificiales mediante la inmersión de premolares y molares sanos en una solución de ácido láctico $0,1 \mathrm{M}(\mathrm{pH} 4,5)$ mantenidos a $37^{\circ} \mathrm{C}$, durante 8 semanas. Posteriormente, las muestras fueron distribuidas en 3 grupos y se infiltraron con una de las resinas estudiadas; grupo A: ICON; B: XP-Bond y grupo C: Single Bond 2. Después se realizaron las pruebas de microdureza por indentación con un durómetro, seguido de un proceso de termociclado (1.000 ciclos) y se midió nuevamente la microdureza.

Resultados: Los valores de microdureza (VHN) obtenidos fueron: ICON=106,9 $\pm 37,1$ y postermociclado= $139,13 \pm 55$, XP-Bond 51,01 $\pm 21,8$ y postermociclado $=103,14 \pm 58$ y Single Bond $2=25,4 \pm 5,8$ y postermociclado $=26,6 \pm 3,5$.

Discusión: Posterior al proceso de termociclado (stress térmico) no se encontraron diferencias estadísticamente significativas entre los grupos infiltrados con ICON ${ }^{\circledR}$ y XP-Bond, en cambio estas diferencias sí existían con respecto del grupo tratado con Single Bond 2.

Palabras clave: Caries incipientes artificiales; ICON; infiltración con resinas; microdureza; termociclado.

\section{SUMMARY}

Introduction: Minimally invasive dentistry, the procedure called Caries Infiltration seeks to stop the progression of incipient caries by sealing the enamel microporosities. This treatment is performed with the application of low viscosity resins. The objective of this work was to evaluate the Microhardness of artificial white-spot lesions infiltrated with low viscosity resins before and after Thermocycling.

Methodology: It caused artificial caries by immersion of healthy premolars and molars in a solution of $0,1 \mathrm{M}$ lactic acid $\left(\mathrm{pH} \mathrm{4,5)}\right.$ maintained at $37^{\circ} \mathrm{C}$ for 8 weeks. Subsequently, samples were divided into 3 groups and infiltrated with studied resins; Group A: ICON, B: XP-Bond and group C: Single Bond 2. Later, the Microhardness tests were executed by indentation with a Durometer, followed by a process of Thermocycling (1000 cycles), and the Microhardness was measured again.

Results: Microhardness (VHN) values obtained were: $\mathrm{ICON}=106.9 \pm 37.1$ and post thermocycling $=139,13 \pm 55$, XP-Bond 51,01 \pm 21.8 and post thermocycling $=103,14 \pm 58$, and Single Bond $2=25.4 \pm 5.8$ and post thermocycling $=26.6 \pm 3.5$.

* Departamento Odontología Integral del Adulto. Facultad de Odontología. Universidad Mayor. Santiago, Chile.

Trabajo adscrito a Proyecto de Investigación parcialmente financiado por Concurso Colgate 2014. 
Discussion: Posterior to thermal cycle (thermal stress) no statistically significant differences were found between groups infiltrated with ICON ${ }^{\circledast}$ and XP-Bond, instead these differences do exist with respect to the group treated with Single Bond 2.

Key words: Artificial white-spot lesions; ICON; resin infiltration; microhardness; thermocycling.

Fecha de recepción: 24 de marzo de 2015.

Aceptado para publicación: 5 de mayo de 2015.

Zamorano Pino X, Valenzuela Aránguiz V, Vial Prado C, Vidal Tardón M. Microdureza de caries incipientes artificiales infiltradas con resinas de baja viscosidad antes y después del termociclado. Av. Odontoestomatol 2015; 31 (6): 371-378.

\section{INTRODUCCIÓN}

Actualmente se considera que la lesión de caries es el resultado de numerosos episodios de desmineralización y remineralización, en lugar de un proceso único de desmineralización (1) Cuando el equilibrio se inclina hacia la pérdida de minerales se pueden evidenciar los primeros signos clínicos de la caries; la manifestación más temprana de esta lesión es la "mancha blanca", la cual se visualiza como un esmalte de apariencia opaca y blanquecina debido a la disminución de la translucidez (2). Histológicamente, esta lesión inicial presenta una pérdida de minerales de un $30 \%$ que se manifiestan como poros y que se extienden en la subsuperficie del esmalte, es decir, esta zona es porosa y permeable. En cambio, la capa superficial se caracteriza por estar relativamente intacta $(3,4)$.

A modo de respuesta hacia los hechos antes mencionados, el enfoque de una odontología restauradora se ha transformado hacia una odontología mínimamente invasiva, cuya finalidad es mantener al diente sano y funcional, evitando realizar procedimientos invasivos. Esto se puede lograr a través del uso de materiales diseñados específicamente para el tratamiento de caries incipientes denominados resinas infiltrantes, cuyo propósito es detener el avance de las lesiones cariosas en etapas donde la terapia remineralizadora no es suficiente ni eficiente (5).

Las resinas infiltrantes son monómeros de baja viscosidad con un alto coeficiente de penetración, los cuales después de polimerizados constituyen una barrera bajo la superficie adamantina que inhibirían la progresión de la lesión, fenómeno que ha sido reportado en algunos estudios clínicos. El uso de estos polímeros ha sido clasificado como terapia micro invasiva, ya que impiden la destrucción de tejido sano, pero a la vez necesitan acondicionamiento del esmalte previo a su aplicación $(5,6)$.

En 2007, Paris y Meyer-Lueckel desarrollaron una línea investigativa utilizando adhesivos convencionales con modificaciones en sus porcentajes de BISGMA, TEGDMA, HEMA y etanol, con la finalidad de mejorar la capacidad de penetración del material (7). El resultado de estas investigaciones fue la creación de una resina infiltrante comercializada con el nombre de ICON ${ }^{\circledR}$ (DMG-AMERICA), diseñado específicamente para el tratamiento de caries incipientes.

Sin embargo, no existe suficiente evidencia científica independiente para promover su uso ya que la mayoría de los estudios fueron realizados por el mismo grupo de investigadores que crearon este sistema de resinas infiltrantes.

En términos de durabilidad, una de las características relevantes de los materiales restauradores en base a resinas es la dureza superficial que logran una vez polimerizados, la cual se define como la resistencia a la deformación permanente. Conjuntamente con lo señalado, los estudios in vitro deberían considerar las condiciones orales, donde los materiales dentales son sometidos a esfuerzos mecánicos y cambios extremos de temperatura, entre otras circunstancias que atentan contra la longevidad y estabilidad en la cavidad oral. 
Por tanto, el propósito de este trabajo fue evaluar y comparar la Microdureza superficial obtenida al infiltrar lesiones incipientes creadas artificialmente, con la resina infiltrante ICON ${ }^{\circledast}$ y con dos sistemas adhesivos convencionales, antes y después de ser sometidas al proceso de termociclado.

\section{MATERIAL Y MÉTODO}

Se utilizaron 70 dientes sanos (premolares y terceros molares con indicación de exodoncia) los que fueron higienizados con escobilla más pasta profiláctica y conservados en agua destilada a temperatura ambiente hasta su utilización. A estas piezas se les seccionaron las raíces, dejando sólo la corona y la cámara pulpar se selló con cemento de vidrio ionómero. Posteriormente, se eligieron 60 de estas coronas al azar y se les aplicó barniz de uñas transparente en toda su superficie, dejando áreas libres de $6 \times 6 \mathrm{~mm}$ en las caras vestibular y palatina o lingual. Luego, fueron sumergidas durante 8 semanas en una solución de ácido láctico $0,1 \mathrm{M}$ a pH 4,5 (estabilizado con $\mathrm{NaOH}$ ) y mantenidas en una estufa a $37^{\circ} \mathrm{C}$. La solución fue reemplazada dos veces por semana, observándolas cada 3 días hasta obtener la formación de una lesión similar a una caries incipiente, bajo el criterio de que fueran visibles con la superficie húmeda (código ICDAS 2).

Una vez terminada la formación de caries artificiales, se cortaron las coronas por la mitad, en sentido mesiodistal, obteniéndose 100 superficies las que se incluyeron en bloques circulares de acrílico que exponían sólo la zona con caries incipiente (Fig. 1). Del mismo modo, se prepararon, además, 20 probetas con esmalte sano.

Las muestras con caries fueron divididas en cuatro grupos de 25 cada uno. Se dejó un grupo control con caries sin tratamiento y los grupos A, B y C correspondían a caries artificiales infiltradas con alguna de las resinas de este estudio, de la siguiente forma: Grupo A: resina infiltrante ICON, Grupo B: adhesivo XP-Bond y Grupo C: adhesivo Single Bond 2. Además se consideró control el otro grupo de las 20 muestras de esmalte sano.

El procedimiento con el cual se aplicó el ICON fue el indicado por el fabricante: Se grabó la superficie con

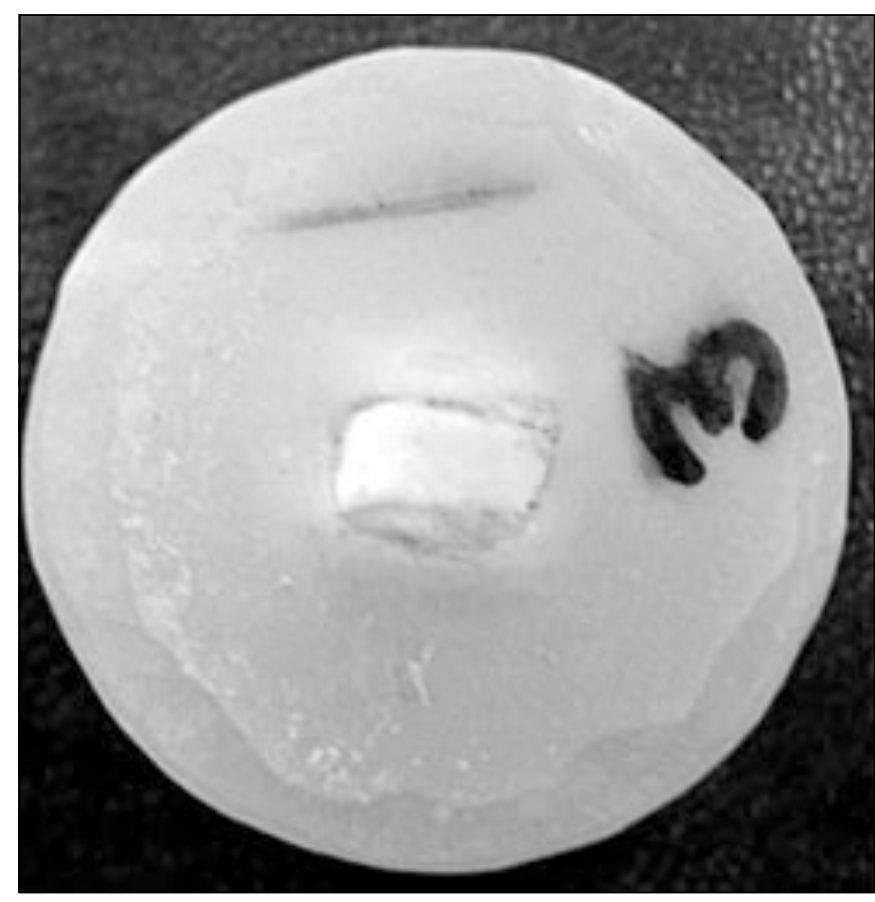

Fig. 1. Muestra de caries incipiente artificial montada en el bloque de acrílico.

ácido clorhídrico al 15\% (Icon Etch) por 2 minutos, se lavó abundantemente por 30 segundos, seguidamente se aplicó etanol (Icon Dry) por 30 segundos, y al finalizar se secó la superficie por 2 segundos con aire. Luego se aplicó la resina infiltrante (Icon Infiltrant) por 3 minutos, se removió el exceso con aire por 3 segundos y se polimerizó por 40 segundos. Por último, se aplicó una segunda capa de Icon Infiltrant por 1 minuto y se polimerizó nuevamente durante 40 segundos (Elipar 2.500, $650 \mathrm{~mW} / \mathrm{cm}^{2}$, 3M-ESPE).

En las muestras que fueron tratadas con los adhesivos convencionales XP-Bond o Single Bond 2, se utilizó el siguiente procedimiento: se grabó la superficie con ácido ortofosfórico al $37 \%$ por 30 segundos y se lavó abundantemente durante 30 segundos. Posteriormente se secó la superficie con aire por 3 segundos y se aplicó una capa de adhesivo frotando suavemente con un microbrush durante 1 minuto. Luego se removieron los excesos con aire por 3 segundos y se colocó una segunda capa de adhesivo para finalizar polimerizando por 40 segundos (Elipar 2.500, $650 \mathrm{~mW} / \mathrm{cm}^{2}, 3 \mathrm{M}$ ESPE). 
Una vez preparadas todas las muestras, se midió la microdureza superficial del esmalte con un durómetro (modelo Leitz Miniload 2, Microhardness Tester), operado por personal especializado en el uso de esta máquina. Las pruebas consistieron en tres micro indentaciones en cada muestra con una punta de diamante bajo una carga de 50 Pounds por 27 segundos, lo cual generó una hendidura de forma cuadrada con 2 diagonales en la superficie de cada probeta (Fig. 2). Estas diagonales se midieron y se sacó el promedio de ambas para cada muestra (Fig. 3). El valor obtenido fue trasladado a una tabla de conversión proporcionada por el fabricante que entregó la microdureza en unidades Vickers (VHN) equivalentes a $\mathrm{Kp} / \mathrm{mm}^{2}$.

Posteriormente las muestras de los grupos A, B y C fueron sometidas a un proceso de termociclado (1.000 ciclos), que consistió en la inmersión de las probetas en dos estanques con agua, uno a $10^{\circ} \mathrm{C} \pm 5^{\circ} \mathrm{C}$ y otro a $70^{\circ} \mathrm{C} \pm 5^{\circ} \mathrm{C}$ por 30 segundos en cada estanque y 10 segundos a temperatura ambiente. Finalizado este proceso, las muestras fueron sometidas nuevamente a pruebas de microdureza.

Estos nuevos valores fueron tabulados y comparados con los obtenidos inicialmente, los que se ana-

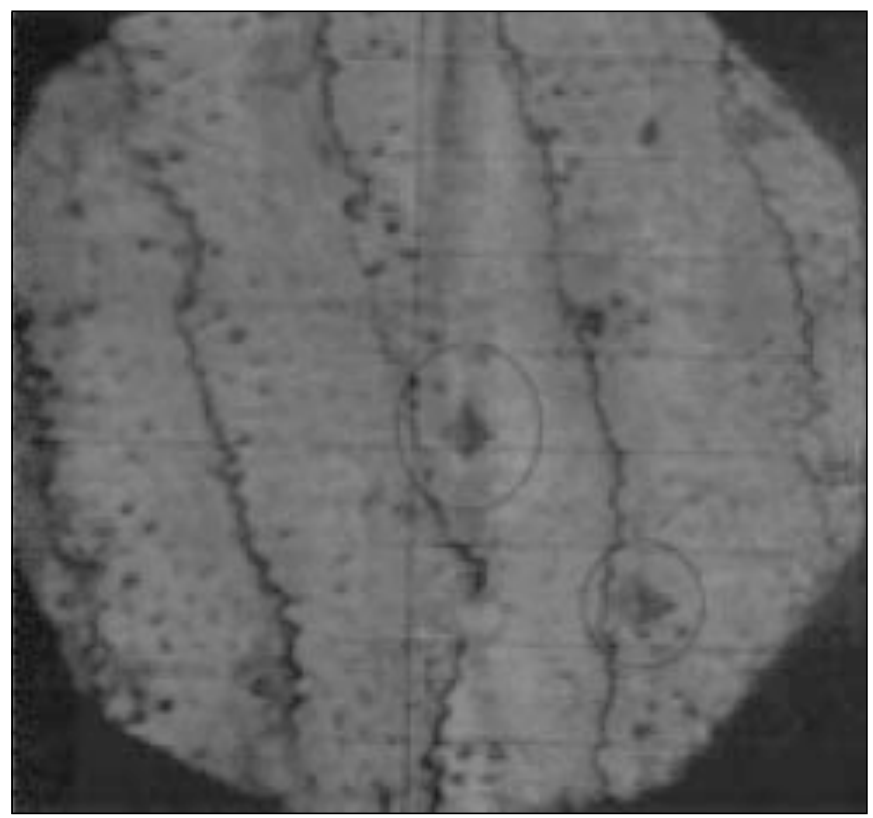

Fig. 2. Imagen microscópica de la indentación obtenida con el durómetro.

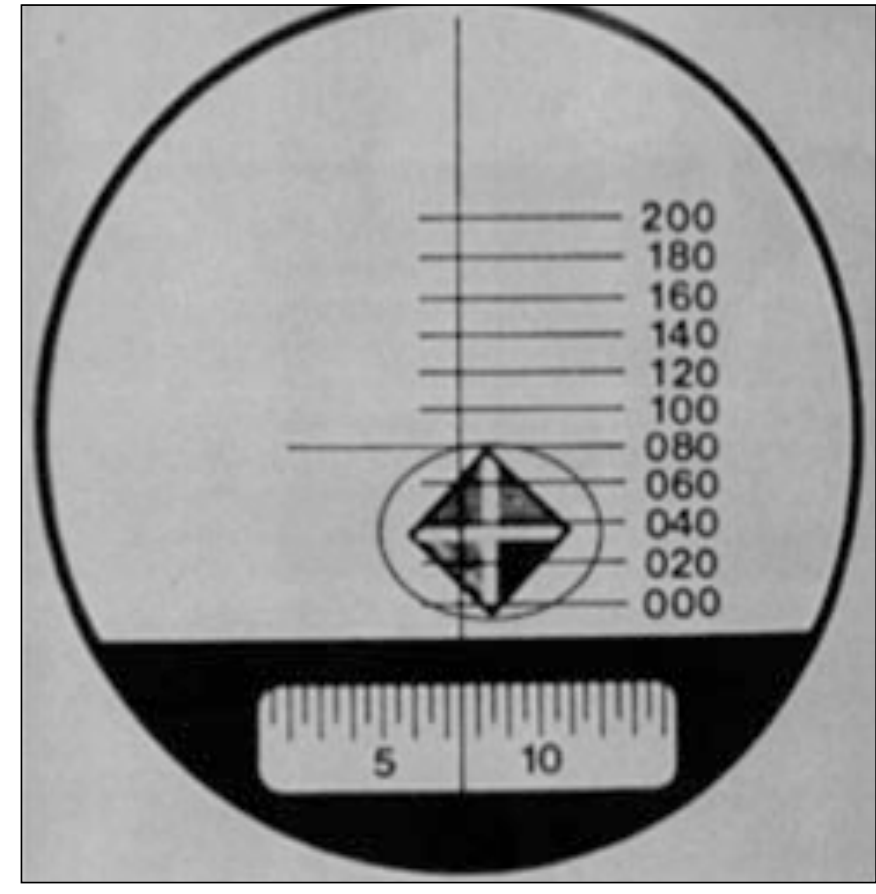

Fig. 3. Imagen referencial de la medición de diagonales obtenidas en la indentación con el durómetro.

lizaron mediante el software Stata/IC 13.1 aplicando los estadísticos de ANOVA, test de Scheffé y test de Student de términos pareados. Para todos los estadísticos se consideró que existían diferencias significativas cuando $\mathrm{p}<0,05$.

\section{RESULTADOS}

Los datos obtenidos de las pruebas de microdureza para los grupos en estudio previo al termociclado se pueden observar en la Tabla 1, la distribución de las muestras se aprecia en el Gráfico 1 y posteriormente se realizaron los test estadísticos correspondientes.

Según este gráfico los datos obtenidos presentan distribución normal. Sin embargo, el promedio de cada grupo y su correspondiente DS indican que existen diferencias significativas entre ellos. ANOVA $\mathrm{F}=80,21 ; \mathrm{p}<0,05$.

Al realizar el test de Scheffé, se observaron diferencias significativas entre el grupo de Esmalte Sano con todos los grupos. El grupo de Caries Incipiente demostró no tener diferencias significativas con los 


\begin{tabular}{|l|c|c|}
\hline \multicolumn{3}{|c|}{ TABLA 1.- VALORES MICRODUREZA } \\
VICKERS (VHN) ANTES DEL TERMOCICLADO \\
\hline Grupo & N & X \pm DS \\
\hline ICON (A) & 22 & $106,9 \pm 37,7$ \\
XP-Bond (B) & 24 & $51,1 \pm 21,8$ \\
\hline Single Bond 2 (C) & 23 & $25,4 \pm 5,8$ \\
Caries incipiente & 20 & $67,2 \pm 24$ \\
\hline Esmalte sano & 18 & $340,7 \pm 136$ \\
\hline
\end{tabular}

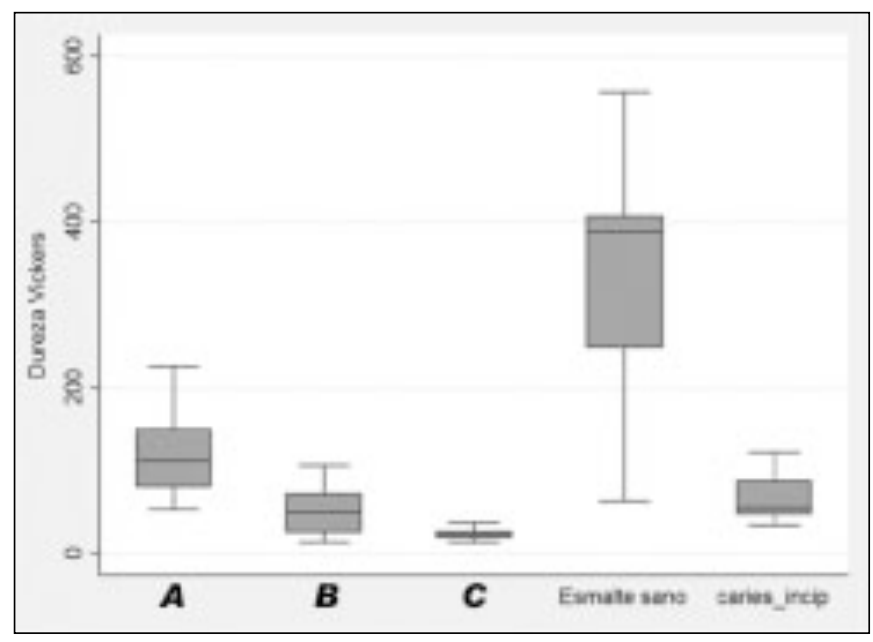

Gráf. 1. Distribución de datos obtenidos, con sus promedios y DS.

\begin{tabular}{|c|c|c|}
\hline \multicolumn{3}{|c|}{$\begin{array}{c}\text { TABLA 2.- VALORES MICRODUREZA } \\
\text { VICKERS (VHN) DESPUÉS DEL } \\
\text { TERMOCICLADO DE LAS RESINAS DE BAJA } \\
\text { VISCOSIDAD }\end{array}$} \\
\hline Grupo & $\mathbf{N}$ & $\mathrm{X} \pm \mathrm{DS}$ \\
\hline $\operatorname{ICON}(\mathrm{A})$ & 20 & $139,1 \pm 54,9$ \\
\hline XP-Bond (B) & 20 & $103,1 \pm 58,2$ \\
\hline Single Bond 2 (C) & 20 & $26,6 \pm 3,5$ \\
\hline
\end{tabular}

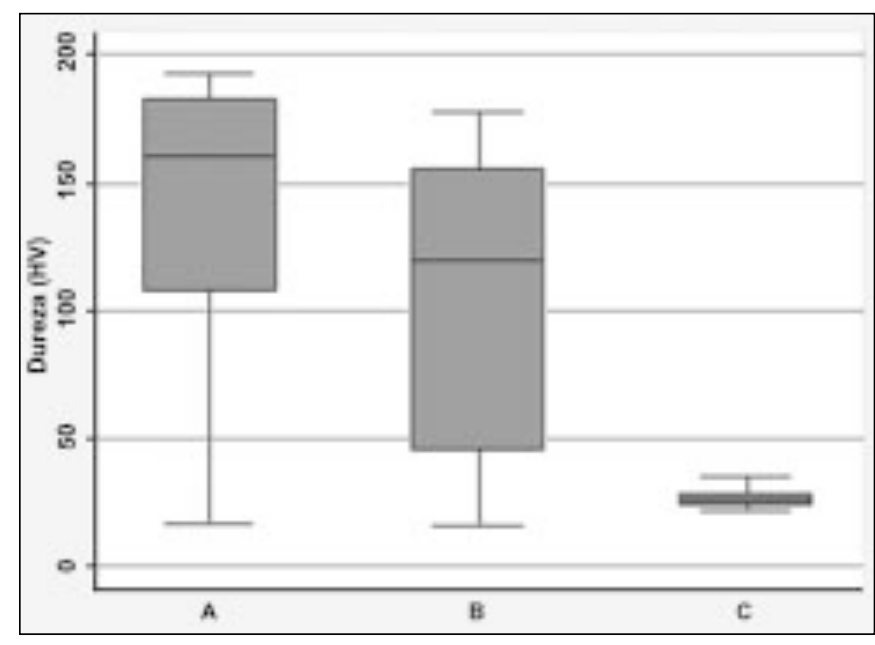

Gráf. 2. Distribución de valores de microdureza Vickers (VHN) después del termociclado de los grupos infiltrados con resinas. A: ICON; B: XP-Bond; C: Single Bond.

el test de ANOVA y test de Scheffé. Los resultados indican que después del termociclado no existieron diferencias significativas entre el grupo A (ICON) y el grupo B (XP-Bond), por su parte entre los grupos A y $C$ se establecen diferencias significativas. Entre los grupos B y C, las diferencias no son estadísticamente significativas posteriores al termociclado.

Finalmente, para establecer si hubo diferencias significativas entre los grupos antes/después del termociclado se realizó un test de Student de términos pareados. En el Gráfico 3, se puede apreciar la distribución de los valores.

Los resultados indicaron que existen diferencias estadísticamente significativas antes/después del proceso de termociclado en el grupo tratado con ICON ${ }^{\circledR}$ 


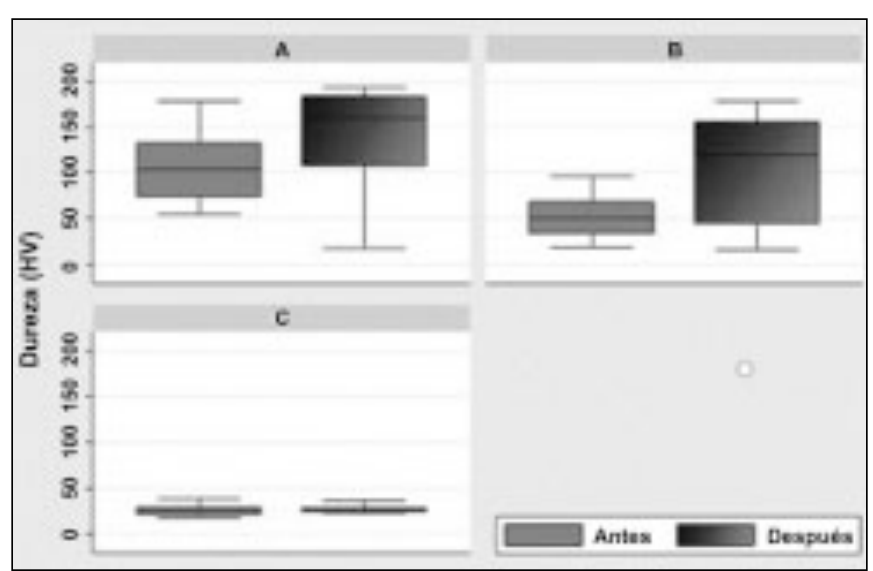

Gráf. 3. Distribución de valores microdureza Vickers (VHN) antes/ después del termociclado. A: ICON, B: XP-Bond, C: Single Bond.

y XP-Bond. Por otra parte, en el grupo tratado con Single Bond no se encontró diferencias estadísticamente significativas antes/después del proceso de termociclado.

\section{DISCUSIÓN}

Según algunos reportes el tratamiento de caries incipientes con resinas infiltrantes ha demostrado ser una opción viable tanto "in vitro" como "in vivo", ya que aumentarían la Microdureza superficial de éstas y conseguirían un sellado de las microporosidades del esmalte subsuperficial, evitando así la progresión de la lesión (8-12).

Los valores de Microdureza de esmalte sano informados por la literatura son de 320 a 400 unidades Vickers y el de caries incipientes son de 20 a 50 VHN (13-15), cifras que coinciden con las encontradas en nuestro estudio. Sin embargo, no encontramos trabajos que informen del comportamiento de estas resinas utilizadas para tratar lesiones de caries incipientes en condiciones de stress o fatiga térmica (sometidos a un termociclado), de ahí la relevancia del presente estudio.

Los valores de microdureza de nuestro estudio son similares a los reportados por Nobrega y col. (10). En caries artificiales infiltradas con ICON ${ }^{\circledR}$ alcanzaron los 92,64 $\pm 72,18$ (VHN), sin embargo, la metodología que utilizaron difiere de la nuestra en cuanto a la formación de caries artificiales, ya que utilizaron ácido acético ( $\mathrm{pH} \mathrm{4,4)} \mathrm{por} 120$ horas, y la carga de indentación fue de $1.000 \mathrm{~g}$, con un reducido número de muestras.

Según nuestros hallazgos, luego del proceso de Termociclado se produjo un incremento significativo en la microdureza de las caries incipientes tratadas con ICON y con XP-Bond, en contraposición a lo señalado por Shantala y Mathew, que concluyeron que el estrés térmico afectaba negativamente las propiedades físicas de los materiales restauradores, incluidas las resinas compuestas (16).

La diferencia en la microdureza de los tres grupos previo al termociclado, se podría explicar por el procedimiento de aplicación y la composición química de las resinas. El grupo tratado con ICON utiliza ácido clorhídrico (15\%) por 120 segundos como acondicionamiento previo en esmalte, lo que lograría una mayor penetración que aquellos que usan ácido ortofosfórico (37\%) por 30 segundos (XP-Bond y Single Bond 2). Otra diferencia es el uso de "ICON DRY" (99\% etanol), lo que según sus fabricantes incrementaría la capacidad de penetración del infiltrante por disminución de la viscosidad y el ángulo de contacto, en comparación al secado con aire de los otros grupos $(17,18)$.

Si bien en ambos grupos tratados con adhesivos convencionales el procedimiento de aplicación es el mismo, es probable que los porcentajes de los componentes varíen, lo que les otorga diferentes propiedades físicas. Cabe señalar que el adhesivo Single Bond 2 contiene un porcentaje de nanorrelleno en su composición, lo que también podría afectar su capacidad de penetración.

El aumento de dureza posterior al termociclado que sufrieron los tres grupos se podría explicar por el proceso de polimerización de éstos, el cual se produce mediante radicales libres que varían su grado de conversión entre 35-80\% (19). Estos alcanzarían el máximo nivel de conversión al ser polimerizados, sin presencia de oxígeno, a altas temperaturas y de forma dual (19-21).

Suponemos que la reacción de polimerización realizada a temperatura ambiente y en presencia de oxí- 
geno impide la conversión completa de monómeros de estos sistemas y tras el termociclado, la exposición a altas temperaturas continuas y repetitivas llevaría a estos polímeros a continuar su reacción aumentando su dureza.

Cabe considerar que nuestro estudio está limitado a los cambios de temperatura, el cual no representa de manera fiel los fenómenos presentes en el medio oral donde otras variables fisiológicas y patológicas interactúan influyendo en el comportamiento de los materiales de restauración.

\section{CONCLUSIONES}

El uso de resinas infiltrantes no aumenta de manera significativa la microdureza superficial de caries incipientes artificiales.

Existen diferencias estadísticamente significativas en la microdureza Vickers del grupo tratado con ICON antes y después del termociclado, siendo mayor posterior al termociclado.

Se encontró diferencias estadísticamente significativas en la microdureza Vickers en el grupo tratado con XP-Bond antes y después del termociclado, siendo superior después del termociclado.

Posterior al proceso de termociclado, no se encontró diferencias estadísticamente significativas entre el grupo tratado con ICON y el grupo tratado con XP-Bond.

En el grupo tratado con Single Bond, no hubo diferencias estadísticamente significativas en la microdureza Vickers antes y después del termociclado.

Después del proceso de termociclado se encontró diferencias estadísticamente significativas entre el grupo tratado con Single Bond y los grupos tratados con ICON y XP-Bond.

\section{BIBLIOGRAFÍA}

1. Frencken JE, Peters MC, Manton DJ, Leal SC, Gordan VV. Minimal intervention dentistry for managing dental caries - a review. Int Dent $\mathrm{J}$. 2012;62:223-43.

2. Fejerskov O, Kidd E. Dental Caries: The Disease and Its Clinical Management. 2nd Ed. John Wiley E Sons 2008:209-30

3. Keyes PH, Jordan HV. Factors influencing the initiation, transmission and inhibition of dental caries. In: Mechanismof hard tissue destruction. NY: Academic Press. 1963;261-83.

4. Guerrieri A, Gaucher C, Bonte E, Lasfargues JJ. Minimal intervention dentistry: part 4. Detection and diagnosis of initial caries lesions. Br Dent $\mathrm{J}$. 2012;213:511-7.

5. Lasfargues JJ, Bonte E, Guerrieri A, Fezzani L. Minimal intervention dentistry: Part 6. Caries inhibition by resin infiltration. Br Dent J. 2013; 214(2):53-9.

6. Paris S, Meyer-Lueckel H. Infiltrants Inhibit Progression of Natural Caries Lesions in vitro. J Dent Res. 2010;89:1276-80.

7. Paris S, Meyer-Lueckel H, Cölfen H, Kielbassa AM. Resin infiltration of artificial enamel caries lesions with experimental light curing resins. Dent Mater. 2007;26(4):582-8.

8. Gugnani N, Pandit K, Gupta M, Josan R. Caries infiltration of noncavited white spot lesions: A novel approach for inmediate esthetic improvement. Contemporary Clinical Dentistry. 2012;3:199202.

9. Gutiérrez B, Planells P. Actualización en odontología mínimamente invasiva: remineralización e infiltración de lesiones incipientes de caries. Cient Dent. 2010;7:183-91.

10. Nobrega D, Kaminsky E, Finkelman M, Kugel G. Unique treatment of early caries and white spot lesions. J Dent Res. 2010;89:22-25.

11. Paris S, Hopfenmuller W, Meyer-Lueckel H. Resin Infiltration of Caries Lesions: an Efficacy Randomized Trial. J Dent Res. 2010;89:823-6. 
12. Swift EJ, Heymann HO, Perdigão J. Bonding to enamel and dentin: A brief history and state of the art, 1995.Quintessence Int. 1995;26:95-110.

13. Craig RG. The microhardness of enamel and dentin. J Dent Res 1958;17:661-8.

14. Gunnar Ryge, Donald Foley, Carl Fairhurst. Micro-indentation Hardness. J Dent Res. 1961;40: 1116-26.

15. Paris S, Schwendicke F, Seddig S, Muller W-D, Dorfer C, Meyer-Lueckel H. Micro-hardness and mineral loss of enamel lesions after infiltration with various resins: Influence of infiltrant composition and application frequency in vitro. J Dent. 2013; 41:543-8.

16. Shantala G.S., Mathew X. The effect of thermocycling on fracture toughness and hardness of different core build up materials. IJDR. 2013; 24:653-8.

17. Martignon S, Castiblanco A, Zarta O. Sealing and Infiltration of Initial Approximal Caries Lesions as an Alternative for Operative Treatment. Literature Review. Univ Odontol. 2011;30:51-61.
18. Subramaniam P, Girish Babu KL, Lakhotia D. Evaluation of penetration depth of a commercially available resin infiltrate into artificially created enamel lesions: An in vitro study. J Cons Dentistry. 2014;17:146-9.

19. Souza R, Özcan M, Michida S. Conversion Degree of Indirect Resin Composites and Effect of Thermocycling on Their Physical Properties. J of Prosth. 2010;19:218-25.

20. Craig R.G. Materiales de Odontología Restauradora. 10 ${ }^{\mathrm{a}}$ Ed. Harcourt Brace; 1998:127-36.

21. Rawls R. Polímeros Dentales. Phillips Ciencia de los materiales dentales. $11^{a}$ Ed. Elsevier; 2004: 143-66.

\section{CORRESPONDENCIA}

Dra. Ximena Zamorano Pino

Las Bellotas, 199 of. 75

Providencia

Santiago, Chile

Correo electrónico: doczamor@vtr.net 